\title{
Evaluation of Optical Wireless Technologies in Home Networking: An Analytical Hierarchy Process Approach
}

\author{
G. Dede, T. Kamalakis, and D. Varoutas
}

\begin{abstract}
Home networks (HNs) will play a crucial role in achieving broadband service delivery and enabling the future Internet. Optical wireless $(\mathrm{OW})$ is a promising technology for realizing this vision. This paper presents the main results of a roadmapping effort undertaken within the project ICT-OMEGA concerning the potential of OW HNs. Using the framework of the analytic hierarchy process, five different optical home networking scenarios are identified and ranked. Within this framework, the importance of several economic, social and performance criteria is also evaluated. The obtained results are justified taking into account the technical particularities of each architecture. A sensitivity analysis is also performed to further elaborate on the results.
\end{abstract}

Index Terms-Analytic hierarchy process; Decision making; Optical home networking.

\section{INTRODUCTION}

$\mathbf{H}$ ome networks (HNs) are expected to play an important role in further promoting broadband penetration and enabling the future Internet. In-building networks, for instance, in corporate or academic settings, traditionally tend to have a tenfold higher capacity than their access points to the rest of the telecommunication infrastructure. Fiber-to-the-home (FTTH) promises symmetric access data rates of at least $100 \mathrm{Mb} / \mathrm{s}$ per household [1] and, hence, HNs should support gigabit-per-second data transmission. The distribution of such high data rates at customer premises is a technological challenge. Given the large capacity of optical communications systems in long haul and metropolitan area networks, one could think of using optics in home networks as well [2]. Short range fiber systems (based on either glass or plastic [3] fibers) may be used inside the home to ensure that no bottlenecks will occur in end-to-end HN services. However, one should also bear in mind that optical technologies are faced with a different set of requirements when deployed inside customer premises. For

Manuscript received June 21, 2011; revised September 26, 2011; accepted September 26, 2011; published October 23, 2011 (Doc. ID 149391).

G. Dede (e-mail: gdede@di.uoa.gr) is with the Department of Informatics and Telecommunications, University of Athens, Panepistimiopolis, Ilisia, Athens, GR15784, Greece, and is also with the Department of Informatics and Telematics, Harokopion University of Athens, Harokopou 89, Kallithea, Athens, GR17471, Greece.

T. Kamalakis is with the Department of Informatics and Telematics, Harokopio University, Greece.

D. Varoutas is with the Department of Informatics and Telecommunications, University of Athens, Panepistimiopolis, Ilisia, Athens, GR15784, Greece.

Digital Object Identifier 10.1364/JOCN.3.000850 example, in existing buildings, residents will be eager to avoid new cable installations which may come at an increased cost and discomfort.

Optical wireless (OW) technology is a means of providing large transmission capacities by letting the optical signals propagate directly into free space, thereby relaxing cableinduced constraints [4]. Such systems are already being widely applied in point-to-point outdoor connections in the access network [5]. Recent years have seen a growing interest in indoor applications as well, in both the infrared [6] and the visible portion [7] of the spectrum. The latter technology, also known as visible light communications (VLC), relies on white light emitting diodes (LEDs) which are used to provide illumination and communication simultaneously. The advantages of OW technology include the virtually unlimited available bandwidth, its inherent security (since the electromagnetic field at these frequencies cannot pass through walls) and limited interference with domestic appliances and conventional radio communication systems. In addition, provided that certain eye safety regulations are met, electromagnetic radiation at these wavelengths is safe, since mankind has been exposed to it for centuries, because of the Sun. Multi-gigabit transceivers have already been developed and commercialized for fiber systems. However, since the issue of cost is of paramount importance near the customer premises, low cost components should be used.

Optical wireless technologies are an integral part of the ICT-OMEGA project [8], the objective of which is to develop a user-friendly home area network capable of delivering high-bandwidth services and content at a transmission speed of one gigabit-per-second [9]. Figure 1 shows a typical home network, as envisioned in the OMEGA project. A variety of user devices are connected through the home network. Data are distributed inside the home network through a number of access points which act as network extenders. The project considered many alternative "no-new-wire" technologies such as conventional and $60 \mathrm{GHz}$ radio systems, OW, ultrawideband (UWB) and power-line communications (PLC), each having the merit of not requiring new cable installations. The project successfully demonstrated IR data transmission at $1.25 \mathrm{~Gb} / \mathrm{s}$ [10] and VLC transmission at $100 \mathrm{Mb} / \mathrm{s}$ [11]. These solutions are not commercially available yet, but rather constitute experimental testbed demonstrations. The combination of these two technologies in a single inter-medium access control (MAC) layer, also demonstrated within OMEGA [9], opens up intriguing possibilities for future home networking. 


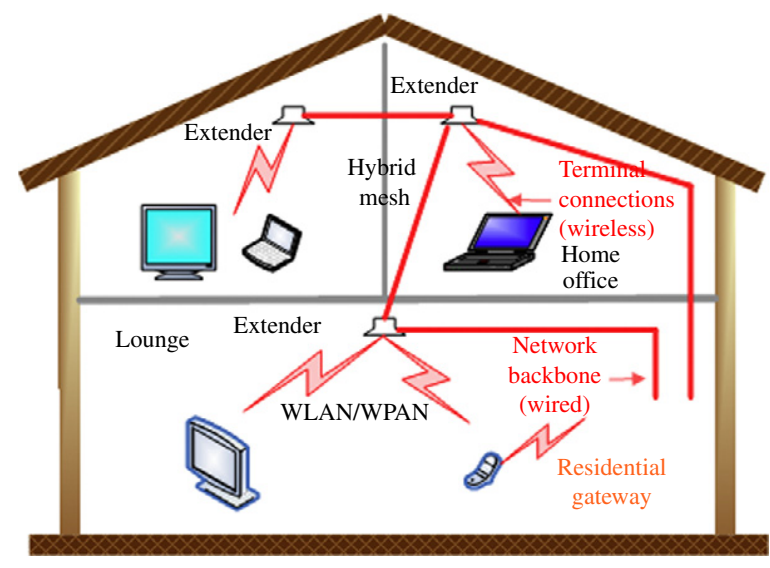

Fig. 1. (Color online) The future home network.
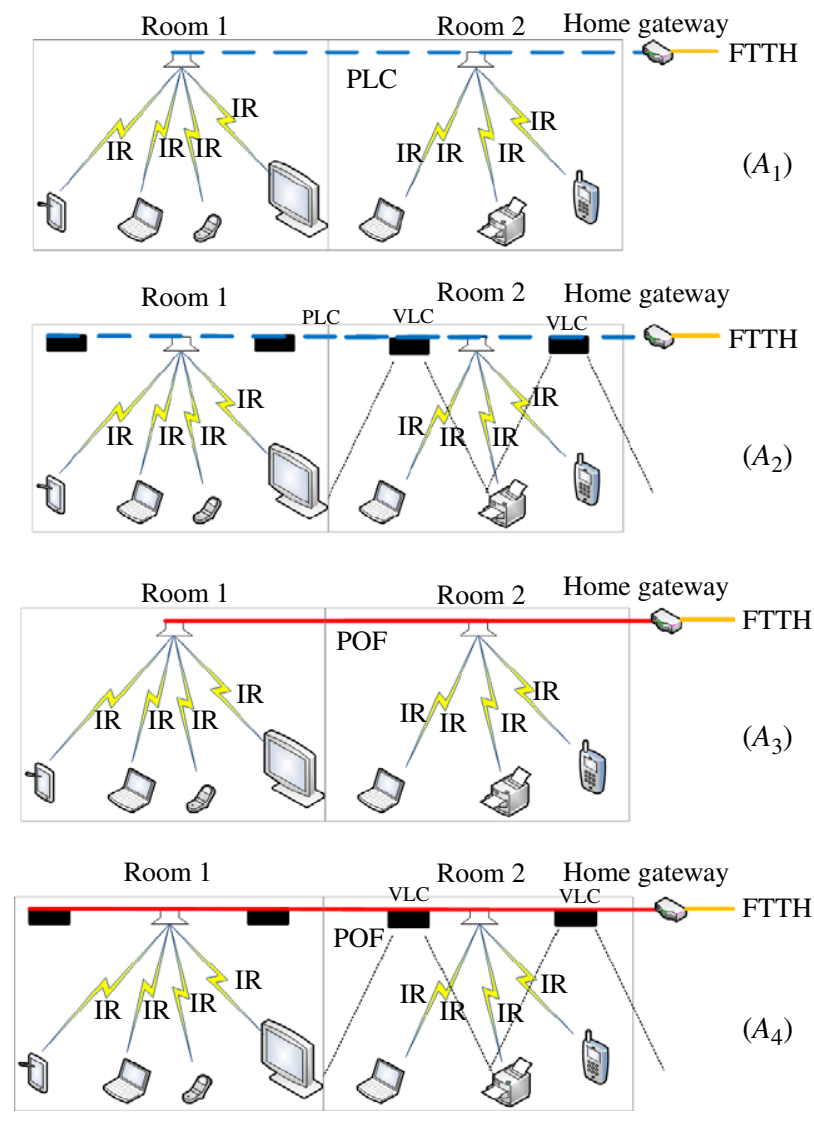

Fig. 2. (Color online) Alternative optical home network scenarios.

The above considerations illustrate that OW may constitute a good candidate for enabling the delivery of many broadband services such as high definition television (HDTV) and Web 2.0 applications including content sharing, online gaming, etc. Unlike conventional radio wireless systems, however, OW is not a mature technology and there are several issues concerning its deployment that remain unclear. One example is the choice of the backbone home network, i.e., the portion of the network that connects optical hotspots to the gateway.
The backbone should be able to support $\mathrm{Gb} / \mathrm{s}$ connectivity or otherwise the data rates provided by the optical hotspots will not be utilized in an efficient manner. PLC can offer an interesting solution [12]. Alternatively, if one is willing to abandon the no-new-wire concept, then optical fibers, either multimode or plastic, can be used for hotspot interconnection. Another issue to resolve is what actual system to use for wireless connections. Do we rely on IR or VLC or both?

The above issues are complicated by the fact that technology penetration depends on a blend of economic, social and performance related criteria [13]. In this paper, we attempt to shed some light on this complex problem, using the framework of the analytical hierarchy process (AHP). The AHP has been used around the world in a wide variety of decision situations, in fields such as government, business, industry, healthcare and education [13-19]. Five alternative deployment scenarios are identified and ranked based on the findings of several carefully designed pairwise comparisons (PWCs). The importance of the various criteria involved in the deployment of the network is evaluated and discussed. The obtained results form a key part of the OMEGA optical wireless roadmap.

The rest of the paper is organized as follows. In Section II, the alternative optical networking scenarios are introduced in more detail along with the basic notions of the AHP methodology. Section III discusses the results obtained by the AHP methodology. A sensitivity analysis in order to validate our results is presented in Section IV. Some concluding remarks are given in Section V.

\section{Deployment Scenario Evaluation}

\section{A. Alternative Deployment Scenarios}

Figure 2 presents four alternative optical wireless deployment scenarios considered in this study. The first one $\left(A_{1}\right)$ relies on bidirectional IR line-of-sight connections and a PLC backbone. The second scenario $\left(A_{2}\right)$ is similar to $A_{1}$ with the exception that VLC lamps are also providing downstream connectivity, enhancing the coverage of the overall system. The VLC and IR subsystems can be combined at a higher network layer such as the MAC layer [9] thereby constituting a hybrid system. Note also that in this scenario VLC is used for downstream data only. The other two architectures, $A_{3}$ and $A_{4}$, are similar to $A_{1}$ and $A_{2}$, respectively, except that a plastic optical fiber (POF) is used at the backbone instead of PLC. Architectures $A_{1}$ and $A_{2}$ have the merit of remaining compatible with the no-new-wires approach. Both PLC and POF are expected to achieve Gb/s connections in the near future. None of the aforementioned wireless alternatives are yet commercially available solutions, but they have been successfully demonstrated in the laboratory. The fifth alternative $\left(A_{5}\right)$, not shown in the figure, is to extend the POF connections right up to the user terminals [20]. No wireless connections are provisioned in this scenario.

\section{B. AHP Methodology}

In order to evaluate the five different home networking architectures discussed in Subsection II.A, one can consider 


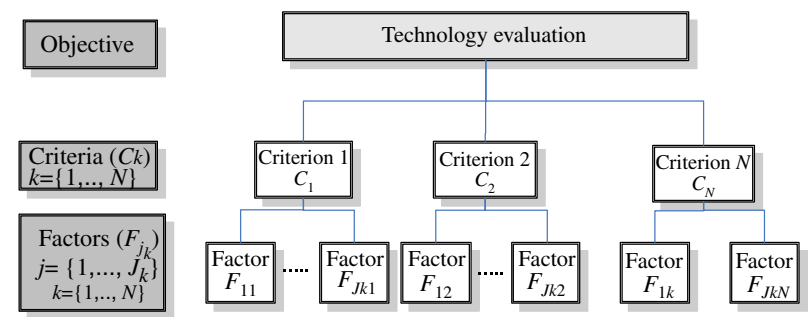

Fig. 3. The hierarchical model for evaluating technologies.

the problem from a decision making point of view and apply the AHP framework, as described in Fig. 3. The AHP adopts a hierarchical form using three conceptual levels. In the first level, the objective for evaluating technologies is defined. In the present case, the overall objective is to produce a roadmap for the optical home/office technologies, rating the five scenarios, and understand the relative importance of various critical issues related to optical home networking.

In the next level, we identify a number of criteria on which our evaluation will be based. Each criterion is an important aspect of the decision making problem and is further identified by its factors at the third level of the hierarchy. A factor is an indicative attribute that characterizes a criterion (e.g., downstream throughput is a factor of performance criterion). We denote the various criteria by $C_{k}$, where $k$ is an integer with $1 \leq k \leq N$, where $N$ is the total number of criteria. The factors of criterion $C_{k}$ are denoted by $F_{j k}$, where $j$ is an integer with $1 \leq j \leq J_{k}$, where $J_{k}$ is the number of factors under criterion $C_{k}$.

In order to rate the alternative technologies, one must first evaluate the weights of the criteria and the factors, denoted by $w_{k}$ and $f_{j k}$, respectively. Toward this end, each expert $m$ performs a series of PWCs by filling out a table containing the upper triangular elements $A_{i j}$ of an $N \times N$ matrix $\mathbf{A}^{(m)}=$ [ $\left.A_{i j}^{(m)}\right]$, which signify the importance of $C_{i}$ compared to $C_{j}$. If, for example, an expert assigns $A_{i j}^{(m)}=60$, then this implies that, according to his point of view, the weight of criterion $C_{i}$ is $60 \%$ compared to the total weight of both criteria, while that of criterion $C_{j}$ is $40 \%$. Using the upper triangular elements of $\mathbf{A}^{(m)}$, a new $N \times N$ matrix $\mathbf{P}^{(m)}=\left[P_{i j}^{(m)}\right]$ is calculated, where $P_{i j}^{(m)}=A_{i j}^{(m)} /\left(100-A_{i j}^{(m)}\right)$ for the upper diagonal elements $(i<j), P_{i j}^{(m)}=1 / P_{j i}^{(m)}$ for the lower diagonal elements $(i>$ $j)$ and the diagonal elements $P_{i i}^{(m)}$ are all set equal to 1 . The elements of $\mathbf{P}^{(m)}$ represent the relative importance of criterion $C_{i}$ compared to $C_{j}$. The criteria weights $w_{k}^{(m)}$ for the $m^{\text {th }}$ expert are obtained by the elements of the eigenvector corresponding to the maximum eigenvalue of $\mathbf{P}^{(m)}$ [21] and are normalized so that $\sum_{k} w_{k}^{(m)}=1$. A similar procedure is followed for the estimation of the weights of the factors $f_{j k}$ of each criterion. Finally, the alternatives are pairwise compared according to each factor and for each alternative $A_{i}$ one obtains the relative scores $S_{i j k}$ under factor $F_{j k}$. The final ranking priorities $T_{i}$ of each alternative are evaluated by multiplying the relative scores $S_{i j k}$ by the overall weight $f_{j k} \cdot w_{k}$ of the corresponding factor as follows:

$$
T_{i}=\sum_{k=1}^{N} \sum_{j=1}^{J_{k}} S_{i j k} f_{j k} w_{k} .
$$

Care should be taken so that the PWC matrices produced by the experts are as consistent as possible. The PWC matrix $\mathbf{P}^{(m)}$ is said to be perfectly consistent if all its elements are of the form $P_{i j}^{(m)}=q_{i}^{(m)} / q_{j}^{(m)}$, where $q_{i}^{(m)}, q_{j}^{(m)}$ are positive real numbers. The consistency ratio $(\mathrm{CR})$ is one measure for consistency and can be readily obtained from the PWC matrices as described in [22]. In our case, the $C R$ values were less than 0.1, which is considered acceptable [23]. Table I summarizes the criteria and factors considered in the surveys. As discussed in the introduction, the alternatives should be ranked taking into account social, economic and performance factors. The factors shown in Table I are similar to those considered in the roadmap of other home network technologies [13] and were identified after consulting the experts of the consortium.

\section{Surveys and Participants}

The number of experts participating in the surveys was equal to 11, which constitutes a sufficient group size according to the literature [24-27]. The experts are members of the OMEGA consortium and participated in the surveys, conducted during a period of 3 months, from September 2010 to November 2010. The experts are employees of various organizations inside the OMEGA project, which constitutes a well balanced blend between industry and academia from many parts of Europe (France, UK, Germany and Greece). Their expertise lies primarily in the field of optical networking technologies. The surveys carried out were the following:

a) Survey $S_{1}$ : Evaluation of criteria and factors.

b) Survey $S_{2}$ : Technology rating (estimation of $S_{i j k}$ ) for the home scenario.

c) Survey $S_{3}$ Technology rating (estimation of $S_{i j k}$ ) for the small office scenario.

It was decided to conduct the technology rating surveys twice, differentiating between the application scenarios. In $S_{2}$ and $S_{3}$ the experts were asked to differentiate between the home and the small office application scenarios. On the other hand, concerning the survey $S_{1}$, the experts pointed out that the criteria and factors would have the same bearing for both the home and small office scenarios. Concerning the technology rating surveys, the experts were asked to consider a short-term time period from 2011 to 2015 .

\section{RESULTS AND DISCUSSION}

\section{A. Weighting of Criteria and Factors}

In this section we discuss the results of the first survey concerning the evaluation of the importance of the criteria and factors that affect the deployment of optical home networks. 
TABLE I

Criteria and Factors for the All Optical House/OfFice Roadmap

\begin{tabular}{|c|c|c|}
\hline Criteria-Factors & Description & $f_{j k}(\%)$ \\
\hline \multicolumn{3}{|c|}{$C_{1}:$ Inter-room Performance $\left(w_{1}=23.90 \%\right)$} \\
\hline$F_{11:}$ Reach & $\begin{array}{l}\text { The maximum transmitter/receiver distance that can allow maximum PHY downstream } \\
\text { throughput }\end{array}$ & 21.6 \\
\hline$F_{12}$ : Coverage & The percentage of a typical room (e.g., FT showroom area) that can be covered by a single system & 27.1 \\
\hline $\begin{array}{l}F_{13} \text { : Downstream } \\
\text { throughput }\end{array}$ & Maximum PHY-throughput that can be provided in the downstream direction & 31.1 \\
\hline$F_{14:}$ Upstream throughput & Maximum PHY-throughput that can be provided in the upstream direction & 20.2 \\
\hline \multicolumn{3}{|c|}{$C_{2}:$ Backbone Performance $\left(w_{2}=20.38 \%\right)$} \\
\hline$F_{21:}$ Reach & The maximum wire length that can allow maximum PHY downstream throughput & 36.2 \\
\hline $\begin{array}{l}F_{22:} \text { Downstream } \\
\text { throughput }\end{array}$ & $\begin{array}{l}\text { Maximum PHY-throughput that can be provided in the downstream direction for backbone } \\
\text { connections }\end{array}$ & 38.7 \\
\hline$F_{23:}$ Upstream throughput & $\begin{array}{l}\text { Maximum PHY-throughput that can be provided in the upstream direction for backbone } \\
\text { connections }\end{array}$ & 25.1 \\
\hline \multicolumn{3}{|c|}{$C_{3}:$ Economic $\left(w_{3}=24.48 \%\right)$} \\
\hline $\begin{array}{l}F_{31:} \text { Cost of installation } \\
\text { (labor) }\end{array}$ & $\begin{array}{l}\text { The labor cost required to install the equipment in a typical ( } 2 \text { room, bedroom + living room) } \\
\text { apartment including backbone }\end{array}$ & 27.8 \\
\hline$F_{32:}$ Cost of equipment & $\begin{array}{l}\text { The total cost of the equipment required to provide connections inside a typical ( } 2 \text { room, bedroom } \\
+ \text { living room) apartment }\end{array}$ & 39.9 \\
\hline$F_{33:}$ Maintenance cost & $\begin{array}{l}\text { Annual cost required to maintain the installed equipment (including backbone equipment and the } \\
\text { power bill) }\end{array}$ & 32.3 \\
\hline \multicolumn{3}{|c|}{$C_{4}:$ Social Acceptance $\left(w_{4}=31.24 \%\right)$} \\
\hline$F_{41:}$ Health issues & Are health issues important? (meeting radiation exposure, eye safety, skin safety, etc. standards) & 24.3 \\
\hline $\begin{array}{l}F_{42:} \text { Home integration with } \\
\text { no-new-wires/design }\end{array}$ & $\begin{array}{l}\text { Is it necessary to install new wires in the house? How well do the network devices fit with the } \\
\text { overall household decoration? }\end{array}$ & 20.1 \\
\hline$F_{43}$. Usability & How difficult is it to set up and manage the network from the average user's point of view? & 25.9 \\
\hline $\begin{array}{l}F_{44:} \text { Compatibility with } \\
\text { legacy systems }\end{array}$ & Is the optical home network compatible with existing networks and home appliances? & 29.7 \\
\hline
\end{tabular}

Table I shows the weights of the criteria and factors calculated by $S_{1}$. The results show that the social acceptance criterion is the most important one to take into account as its weight reaches $31.3 \%$. This is a clear indication that the public should be made aware of the merits of optical home technologies. Although optical fibers have been abundantly installed in core and metropolitan area networks, for many people they look and sound like something exotic, probably because their penetration at customer premises is rather limited. In office buildings, people may be slightly more accustomed to optics, because of optical Ethernet interfaces, usually preferred for high speed layer 2 and 3 switch interconnection. This is not the case in home environments (with the possible exception of IrDA and of course TV remote controls). One approach for stimulating the public's interest could be to promote its merits, i.e., its ultrahigh bandwidth, inherent security and safety, etc. The economic criterion has the second largest weight, emphasizing the need for cost-effectiveness as we move closer and closer to the customer premises. Reducing the cost of terminals and hotspots can have a profound effect on the business prospects of the optical home. People nowadays are accustomed to paying less than $100 €$ to buy an $802.11 \mathrm{n}$ router with $\sim 100 \mathrm{Mb} / \mathrm{s}$ wireless connectivity and will be unwilling to replace their existing networks without understanding the potential benefits. Economic criteria are very important in any decision making process for telecom products. Adding a "Gig" in front of access technologies does not guarantee a market potential: the "Gig" must come at the right price. LEDs could go a long way in reducing the cost of the optical transmitter at the expense of smaller modulation bandwidth and reduced transmitted power. Performance related criteria receive the smallest weight. Interestingly enough, inter-room performance seems more important than backbone performance, probably because in local area networks a large amount of traffic can be destined for nearby devices, i.e., sending data to a printer, connecting a game console and the TV set, etc. The role of the backbone should not be dismissed however. In view of the fact that optical signals generated at a hotspot cannot leave the confines of the room, one must come up with a way of extending the network reach across rooms. Table I shows that with the exception of social acceptance, all other criteria have comparable weights. This suggests that optical home networks should be designed in order to fulfill a number of diverse and possibly conflicting criteria.

It is also interesting to examine the weights of the factors under each criterion. Regarding inter-room performance, the experts seem more concerned about the downstream throughput in view of many popular services where bandwidth requirements are asymmetric (e.g., HDTV, web browsing, video-on-demand, etc.). The role of upstream is not negligible, however, accumulating $\sim 20 \%$ of the overall importance, a reflection of the fact that many emerging applications have symmetric bandwidth requirements (Web 2.0, content sharing, online gaming, etc.). Coverage seems the second most important issue, accumulating a weight of $\sim 27 \%$. This is a key issue for OW technologies, especially for line-of-sight systems where there is a trade-off between coverage and received power. Given the limitations in transmitted power (because of eye safety and cost issues), one should look for schemes to further enhance the power budget and guarantee sufficient signal reception in all corners of the room. Coherent detection [28] can be used to improve receiver sensitivity by several $\mathrm{dBs}$ and should be explored in future systems. Multiple input multiple output (MIMO) techniques [29], although not particularly effective in direct detection systems, could also provide increased capacity since the coherent optical channel resembles radio channels in many ways. Using multiple transmitters, each pointing to a different position in the room, is a first step for improving the coverage in IR systems. 
VLC systems, on the other hand, have inherently excellent coverage characteristics as there may be many LED lamps in the ceiling of a room. Inspired by the fact that IR LEDs usually have a much larger bandwidth than their visible counterparts, one could also envision a hybrid IR/visible system where each lamp chip could contain both IR and visible LEDs. The visible LEDs provide illumination and communication while the IR LEDs are used to further enhance the bandwidth of the system. Regarding the weights of the factors of backbone performance, similar conclusions can be drawn to the case of inter-room performance discussed previously. For the backbone infrastructure, there is always a compromise between reach and available bandwidth, and this is a reason why in many corporate or academic settings optical fiber is the medium of choice in order to connect floor switches to the core switch or router. Ten gigabit Ethernet necessitates the use of fiber even for small distances in these scenarios, but installing fibers in residences is much more involved, since one must take into account the severe user discomfort and cost associated with such deployments.

Regarding the economic criterion, great importance is placed on the equipment cost, emphasizing its role as a motivation for potential buyers. Moreover, the maintenance and installation costs are also important issues to consider, taking weights of $28 \%$ and $32 \%$, respectively. This is not surprising as HN equipment should ideally be cheap to install and maintain. It is interesting to note that these economic issues play a critical role in the world of $\mathrm{OW}$, which may require the installation of multiple hotspots on the ceilings of rooms. From a technoeconomic point of view, the cost directly depends on the volume of production [30]. If the demand for OW systems is high, prices will eventually fall as a consequence of mass production.

Regarding the weights of the factors for the social acceptance criterion, compatibility with legacy systems seems to take precedence over the other issues, with a weight of $30 \%$. This is an indication that the experts tend to think that the adoption of future optical home networks demands compatibility with previous legacy systems and other home appliances which have already been installed in the domestic environment. The results also indicate that future optical home networks should not interfere with any legacy network already installed. In fact, using transceivers operating at different wavelengths, it is possible to envision multiple OW local network connections simultaneously supported inside the same room without any bandwidth reduction due to interference effects. Moreover, the experts seem to highly prioritize health issues, reflecting the great public concern on this issue. PLC and POF wired connections should not pose significant health risk to human tissue. It is also interesting to note that IR OW technologies are considered rather harmless as long as they comply with eye safety standards. The growing public concern may hence provide leverage toward OW solutions, especially in hospitals, nursing homes, etc. Furthermore, the experts seem to care a lot about the usability issue, in order that the devices in future optical home networks be as plug and play as possible. This is not surprising since the widespread adoption of future optical networks will greatly depend on the layman's ability to set up and control the network infrastructure in the domestic environment. Home integration with no-new-wires/design has the lowest value of importance, probably because there are still many important technological matters to be resolved in order to provide reliable and cost-effective network functionalities. In light of this, one clearly understands that future optical networks should intrude on the domestic environment as little as possible: unlike working environments, people are much less inclined to let new wires be installed in their homes. This raises an important concern since, although optical fibers (plastic and multimode) would provide the ultimate solution in terms of bandwidth and coverage in new buildings, wireless and PLC solutions are more appropriate in older buildings where the residents are not willing to disturb their environment and install new cables.

\section{B. Scoring the Alternatives}

In this section we consider the results of PWC for the surveys $S_{2}$ and $S_{3}$ concerning the rating of the alternative scenarios in terms of specific factors for both the home and office application scenarios. Moreover, the final ranking priorities of the five alternatives evaluated through Eq. (1) are presented and further discussed, based on the weights of the criteria and the factors estimated in the previous sections and the PWC of surveys $S_{2}$ and $S_{3}$.

Table II shows the relative scores obtained for each architecture. Regarding the health factor, the all-POF architecture takes a small precedence, probably because there are no wireless connections which must conform to eye and skin safety standards while optical power requirements are lower since the signal propagates inside the confines of the fiber. Regarding the usability and compatibility factors of the social criterion, one can see no clear precedence of any single architecture. Such similarities are not surprising since all architectures rely on optoelectronics to some extent or another. Referring to the fourth factor of this criterion, i.e., integration to the home environment, PLC-based solutions take greater precedence over POF because of the fact that in the former case no new wires need to be installed. PLC backbones are rated higher in the home rather than the office scenario. The all-POF scenario seems to score rather low in both application scenarios since it may lead to cable jargon, unlike the wireless alternatives, and limit terminal mobility. Interestingly enough, the hybridization of VLC and IR seems to complicate things compared to a stand-alone IR hotspot, especially in the office scenario. Concerning the economic aspects of the five architectures, no single solution holds the Holy Grail in terms of equipment cost, although experts seem to believe that PLC along with a hybrid VLC and IR would be the most cost effective, probably because no sophisticated IR transmitters need to be placed in the ceiling since downstream coverage would be largely achieved through VLC lamps, containing cheap illumination LED transmitters. Moreover, the all-POF solution rates higher than all the others in terms of installation cost because the installation of wireless hotspot equipment or VLC lamps may lead to additional required manpower. In addition, the all-POF scenario also rates high in terms of maintenance cost, possibly because the other architectures consist of multiple transceivers in ceilings containing additional sensitive equipment. Considering performance issues and more specifically coverage and reach factors, the all-POF 
TABLE II

Relative Scores of Alternatives for Each FaCtor

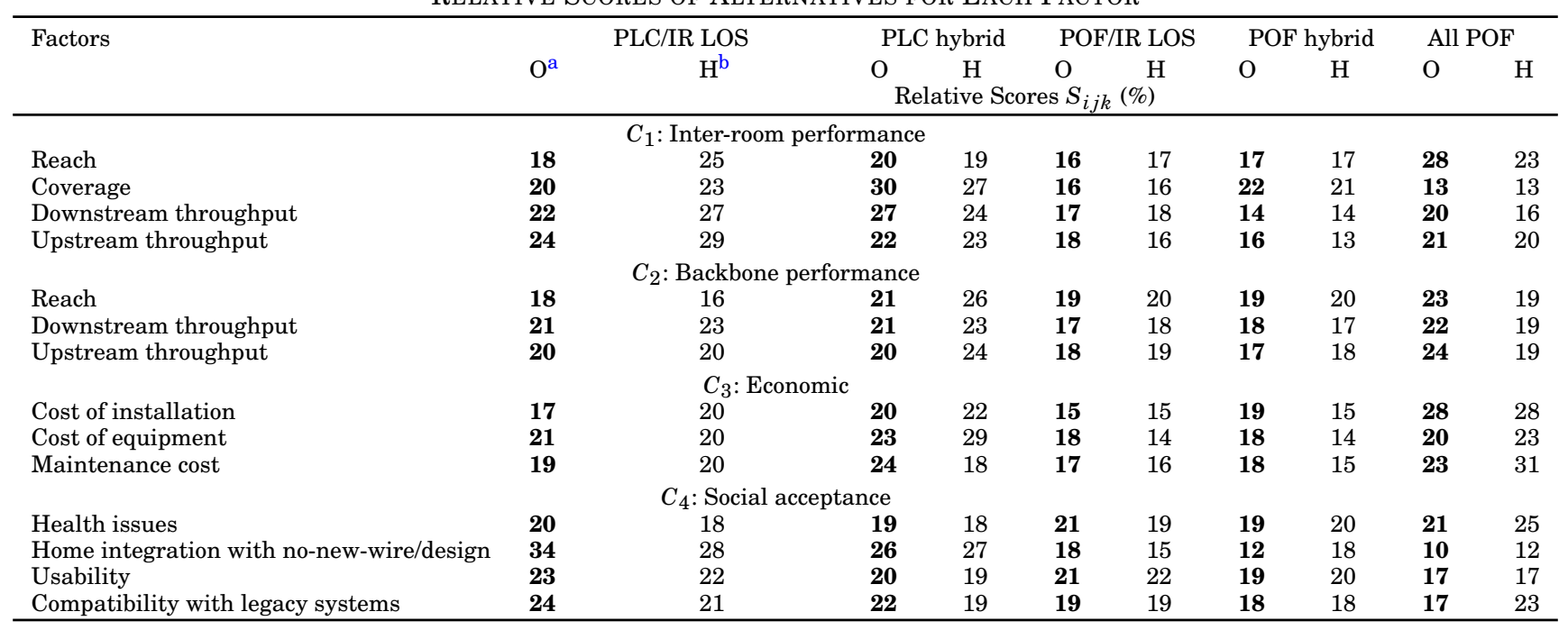

Notes.

a Office scenario

b Home scenario

scenario rates generally slightly better than its wireless counterparts regarding the backbone, which is an indication of the merits of installing plastic fibers regarding network extension. Inside the room, however, the all-POF scenario rates rather low, while hybrid IR/VLC-based scenarios seem to be more favorable. Things are still somewhat unclear regarding the data rate performance of the five architectures, probably because gigabit-per-second is possible in both PLC and POF backbones.

Figure 4(a) shows the ranking priorities of the five alternatives calculated based on the weights of the criteria and the factors estimated in the previous section. Interestingly enough, it seems that both the office and home scenarios are similar, with only small differences in the priorities of the alternatives. The architecture with hybrid VLC/IR hotspots combined with a PLC backbone comes first and is followed closely by the PLC/IR architecture. The all-POF scenario, where no wireless connections are used, comes third, illustrating the potential of plastic fibers in home deployments, especially in the longer term. Hybrid POF and OW scenarios form a third class of alternative. The figure suggests that the vision of the optical home is not yet quite clear and the merits of OW architectures (no additional wires to connect the terminals, mobility, etc.) are somewhat obscured by the level of technological maturity for these solutions.

The results are more definitive for the backbone technology of choice in the case of wireless inter-room connections. One can calculate the priority $T_{\mathrm{PLC}}$ of the PLC backbone solution as the sum of the priorities of the wireless scenarios with PLC backbone, i.e., $T_{\mathrm{PLC}}=T_{1}+T_{2}$. In a similar fashion, one can calculate the priority $T_{\mathrm{POF}}$ of the POF backbone for wireless architectures as $T_{\mathrm{POF}}=T_{3}+T_{4}$. The priorities $T_{\mathrm{PLC}}$ and $T_{\mathrm{POF}}$ are calculated in Fig. $4(\mathrm{~b})$ and PLC communications seem to rate better than plastic fiber for handling backbone traffic. PLC allows for a quick backbone deployment, with data rates that can eventually reach $1 \mathrm{~Gb} / \mathrm{s}$. IEEE P1901 [31] is an IEEE working group developing the global standard for high
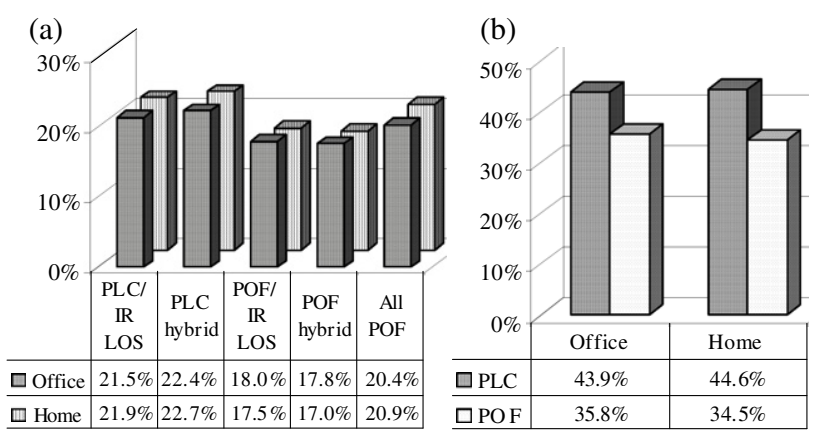

Fig. 4. (a) Alternative priorities for office and home scenarios. (b) Added PLC and POF wireless priorities.

speed PLC. The HomePlugAV2 specification is currently under development and is the prospective next generation for the HomePlug line. Current estimates state that it will operate on a $600 \mathrm{Mb} / \mathrm{s}$ transfer capability. Gb/s transmission over PLC is also demonstrated in OMEGA [12].

\section{SEnsitivity ANALYsis}

In this section we discuss the reliability of the results, given the level of uncertainties involved, by carrying out a sensitivity analysis. Subsection IV.A discusses the impact of changing the value of the weight of a single parameter (e.g., a single criterion weight). In Section IV.B we use Monte Carlo simulation to estimate the effect of introducing random perturbations in all parameters of the decision making process.

\section{A. Changes in a Single Factor or Criterion Weight}

Figures 5(a) to 5(d) illustrate the influence of perturbing the weight $f_{j k}$ of the most important factors by $\Delta f_{j k}$ for the home 


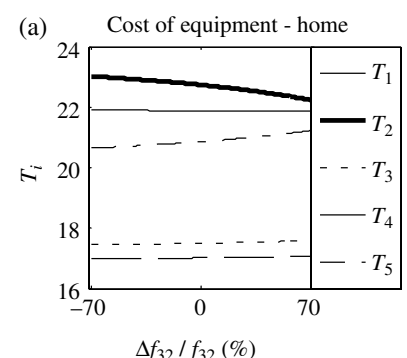

(c) Downstream (inter - room) - home

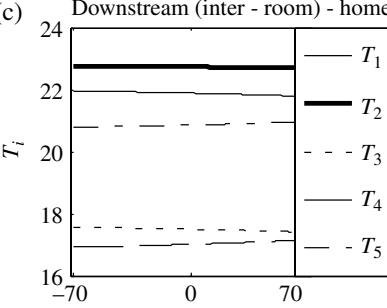

$\Delta f_{13} / f_{13}(\%)$
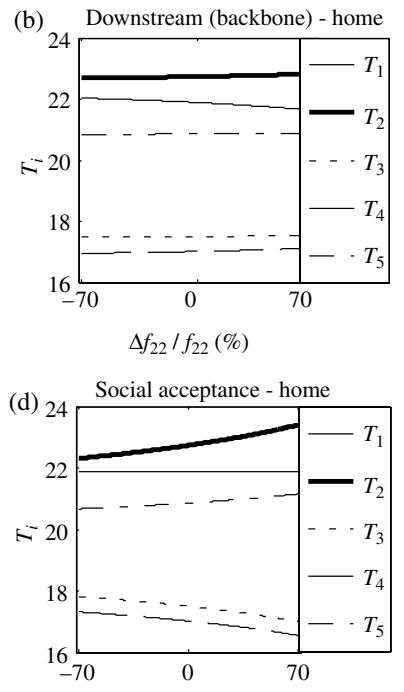

$\Delta w_{4} / w_{4}(\%)$

Fig. 5. Sensitivity to (a) equipment cost, (b) downstream throughput (backbone), (c) downstream throughput (inter-room) and (d) the social acceptance criterion.

scenario. The perturbed weights need to be renormalized so that their sum is equal to unity and consequently changing a single weight will necessarily induce changes in the weights of other factors. Figure 5(a) suggests that changing the weight of the equipment cost, $f_{32}$, affects the final ranking priorities of all five technologies, but not dramatically. The priority $T_{2}$ of $A_{2}$ (PLC and hybrid VLC/IR) approaches the priority $T_{1}$ of $A_{1}$ (PLC/IR). It is interesting to note that the ranking between the various alternatives is preserved even for relative changes as high as $\pm 70 \%$. The situation is pretty much the same if we consider the weights of the downstream throughput factor for both the backbone and the inter-room performance criterion, as shown in Figs. 5(b) and 5(c), respectively. The ranking is again preserved. Similar conclusions are drawn when we perturb the weight $w_{4}$ of the most important criterion, social acceptance, by $\Delta w_{4}$ in the evaluation of the alternatives priorities, as shown in Fig. 5(d).

\section{B. Simultaneous Changes of Criterion/Factor Weights and Relative Scores}

In order to further validate the reliability of the final ranking of the alternatives, Monte Carlo simulations were performed by simultaneously changing more than one parameter. The weights of all criteria were perturbed from $w_{k}$ to $w_{k}\left(1+\Delta W_{k}\right)$, where the perturbations $\Delta W_{k}$ were assumed zero mean, identically distributed, independent random variables uniformly distributed inside $[-s s]$. Such random perturbation may be due to inconsistencies of the PWC matrices [23]. In Fig. 6, we show the probability density functions (PDFs) of the final priorities for the home scenario assuming $s=0.2$ (corresponding to a maximum of $\pm 10 \%$ relative variation). The weights are renormalized so that their sum is equal to 1 . The priorities of the alternative architectures were calculated using $10^{5}$ Monte Carlo iterations. Figure 6 indicates

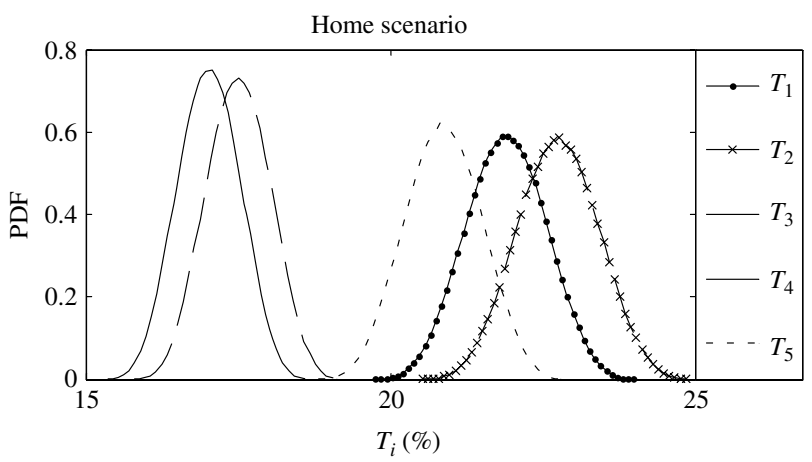

Fig. 6. PDFs of priorities obtained by perturbing the criterion weights.

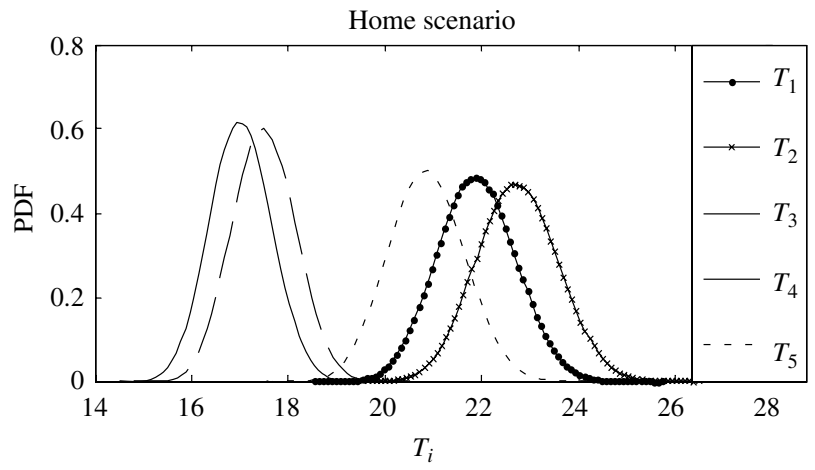

Fig. 7. PDFs of priorities obtained by perturbing the weights of the criteria and factors as well as the technology relative scores.

that the priorities of the technologies have similar shaped PDFs and the priorities for the $A_{1}$ and $A_{2}$ (PLC/IR and PLC/hybrid scenarios) are more prone to uncertainty-induced perturbations (since their PDFs are wider than the rest). The figure also shows that the PDFs of the alternative priorities are centered around the value estimated using the AHP methodology, as depicted in Fig. 4(a), and exhibit a Gaussian-like behavior. Similar conclusions are drawn when the weights, $f_{j k}$, of all the factors are uniformly perturbed.

It is also interesting to calculate the PDFs of the priorities when one perturbs all the parameters (i.e., the $w_{k}, f_{j k}$ and $\left.S_{i j k}\right)$. Figure 7 shows the PDFs of the alternative priorities, while Fig. 8 shows the PDFs of $T_{\mathrm{PLC}}$ and $T_{\mathrm{POF}}$ of the PLC and POF backbone priorities, respectively. These PDFs are again Gaussian in nature.

Figure 9 suggests that the PDFs of the various priorities are overlapping for $s=0.2$. This in turn implies that the ranking of the alternatives may change (a situation referred to as rank reversal). One should, however, bear in mind that the probability of rank reversal for two priorities, say $T_{1}$ and $T_{2}$, cannot be estimated by their individual PDFs $f_{T_{1}}(x)$ and $f_{T_{2}}(y)$ alone since $T_{1}$ and $T_{2}$ can be correlated. To determine the degree of correlation, we compare the combined PDF $f_{T_{1}, T_{2}}(x, y)$ of $T_{1}$ and $T_{2}$ against the product of the individual PDFs $f_{T_{1}}(x) f_{T_{2}}(y)$ in Figs. 9(a) and 9(b), respectively. Monte Carlo simulation was again used for the PDF estimations, assuming uniform perturbations of all parameters with $s=0.2$. 


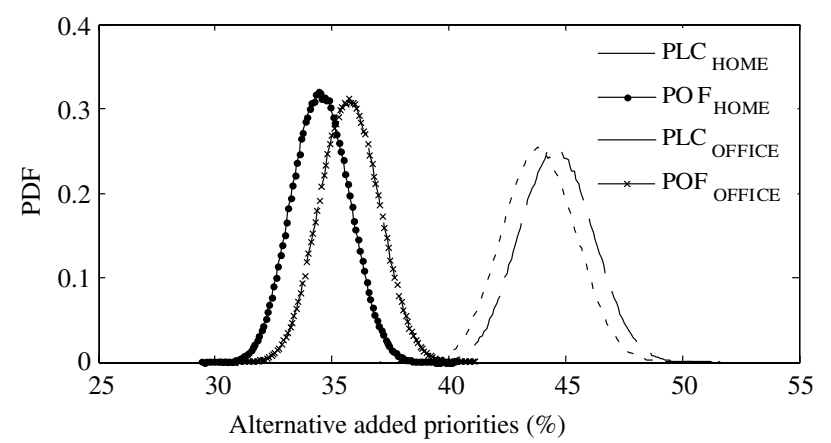

Fig. 8. PDFs of $T_{\mathrm{PLC}}$ and $T_{\mathrm{POF}}$ obtained by perturbing the weights of the criteria and factors as well as the technology relative scores.
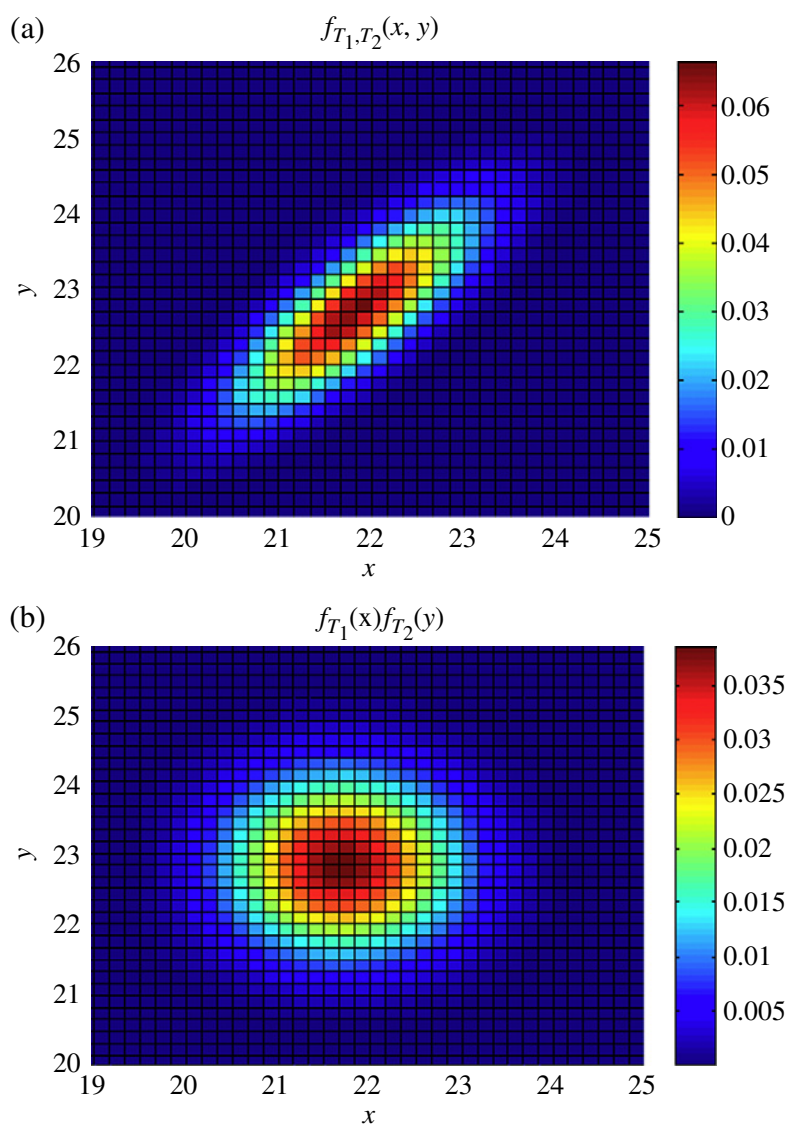

Fig. 9. (Color online) (a) combined PDF of $T_{1}$ and $T_{2}$ and (b) the product of the individual PDFs of $T_{1}$ and $T_{2}$.

The figure illustrates that $T_{1}$ and $T_{2}$ are indeed correlated since $f_{T_{1}, T_{2}}(x, y)$ is quite different from $f_{T_{1}}(x) f_{T_{2}}(y)$. In fact, Fig. 9(a) suggests that $T_{1}$ and $T_{2}$ move in the same direction, i.e., when $T_{1}$ increases, then it is more likely for $T_{2}$ to increase and vice versa. Because of this correlated displacement we expect the probability of rank reversal to be smaller than that implied by the PDF overlap in Fig. 7. The analysis so far was focused on the home scenario, but similar conclusions can be drawn in the case of the office scenario as well.

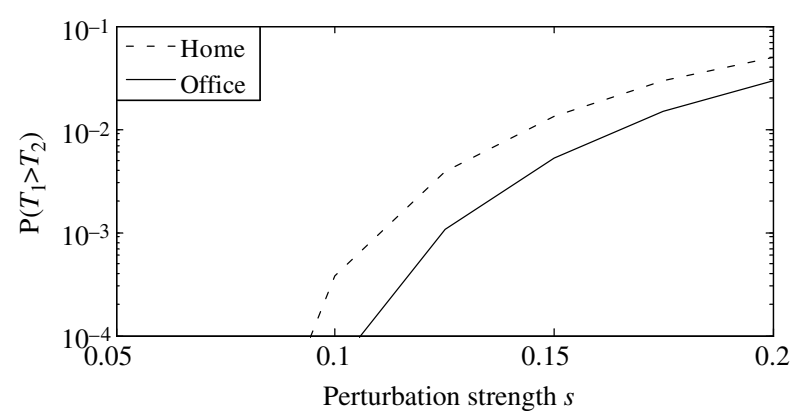

Fig. 10. Probability of rank reversal $P\left(T_{1}>T_{2}\right)$ as a function of the perturbation strength.

Figure 10 shows the probability of rank reversal $P\left(T_{1}>\right.$ $T_{2}$ ) for the priorities of $T_{1}$ and $T_{2}$ for various values of $s$ calculated by Monte Carlo simulation. The figure shows that $P\left(T_{1}>T_{2}\right)$ remains sufficiently low (less than $5 \%$ ) for $s \leq 0.2$ (corresponding to a maximum of $10 \%$ variation in all perturbations) in both the office and the home scenarios. We also calculated the probability of rank reversal in the priorities of the backbone solutions $P\left(T_{\mathrm{POF}}>T_{\mathrm{PLC}}\right)$, which turned out to be negligible (below $10^{-3}$ ) for $s \leq 0.2$. The results provide an indication of the reliability of the AHP results against uncertainties in the PWC carried out by the experts.

\section{Uncertainty in the Pairwise Comparison Matrices}

In an attempt to further ascertain the influence of uncertainties, we carried out Monte Carlo simulations by randomly varying the elements of the PWC matrices used in the estimation of the criteria, factors and alternative relative scores. Assuming the criteria matrices $\mathbf{A}^{(m)}$ filled out by the experts, we estimated the intervals $Q_{i j}=\left[A_{i j}^{(\min )} A_{i j}^{(\max )}\right]$ by calculating $A_{i j}^{(\max )}=\max \left\{A_{i j}^{(m)} \mid 1 \leq m \leq M\right\}$ and $A_{i j}^{(\min )}=$ $\min \left\{A_{i j}^{(m)} \mid 1 \leq m \leq M\right\}$, where $M=11$ is the number of experts in the surveys. In each Monte Carlo iteration, we created $M$ random matrices $\Delta \mathbf{A}^{(m)}=\left[\Delta A_{i j}^{(m)}\right]$ by randomly selecting $\Delta A_{i j}^{(m)}$ from a uniform distribution inside $Q_{i j}$. We also created random PWC matrices for the matrices used to estimate $f_{j k}$ and $S_{i j k}$ and calculated the priorities of the alternatives. Carrying out a large number of iterations $\left(10^{4}\right)$ we estimated the probabilities of rank reversal between the two most prominent alternatives $P\left(T_{1}>T_{2}\right)$ to be equal to $4 \%$ and $6 \%$ for the home and office scenarios, respectively. This is a further indication of the fact that the rankings are not significantly influenced by the uncertainties in the judgments of the experts.

\section{CONCLUSION}

In this paper an evaluation of the potential of $\mathrm{OW}$ technologies for home/office network deployments was carried out. A number of important findings were obtained which must form part of any type of carefully designed roadmap for optical home networking technologies. The first finding concerned the identification and ranking of various factors 
and criteria determining the deployment of such systems. It was made clear that, since home networking systems will be placed at the customer premises, there are many social and economic factors that must be taken into account. Social aspects were shown to be of paramount importance and health issues can provide a serious incentive for installing IR and VLC hotspots which are inherently safe. The results obtained by the surveys were justified taking into account the nature of the optical wireless systems. Next, we identified and ranked five architecture scenarios, consisting of a blend of IR, VLC, POF and PLC systems. These alternatives were evaluated using the AHP framework. The results suggest that a combination of VLC and IR hotspots, along with a PLC backbone, provides the most favorable option, but is closely followed by IR hotspots connected with a PLC backbone. In any case, the results clearly indicated the advantages of a PLC backbone in terms of ease of installation in older buildings. The ranking results were also further elaborated using sensitivity analysis and Monte Carlo simulation. It was found that under uncertainty the priorities of the alternatives are correlated and this correlation reduces the probability of rank reversal. The hybrid VLC/IR with PLC backbone solution is almost never surpassed by the IR PLC alternative, even if all parameters are randomly perturbed by $\pm 10 \%$.

The paper provided a framework to identify factors that could speed up or impede the deployment of optical communication technologies in the home network. It is the hope of the authors that it will constitute a first step in bridging the gap between the important research work carried out in the field and the socio-economic requirements that will guarantee the business prospects of their wide scale deployment.

\section{ACKNOWLEDGMENTS}

The research leading to these results has received funding from the European Community's Seventh Framework Programme FP7/2007-2013 under grant agreement no. 213311, also referred as OMEGA. The authors would like to thank the experts who participated in the aforementioned surveys as well as the people working on the Work package 4 of ICT-OMEGA. Any opinions expressed in the paper may not compeletly reflect the point-of-view of all OMEGA consortium members.

\section{REFERENCES}

[1] P. W. Shumate, "Fiber-to-the-home: 1977-2007," J. Lightwave Technol., vol. 26, pp. 1093-1103, 2008.

[2] R. Gaudino, D. Cardenas, M. Bellec, B. Charbonnier, N. Evanno, P. Guignard, S. Meyer, A. Pizzinat, I. Mollers, and D. Jager, "Perspective in next-generation home networks: Toward optical solutions?" IEEE Commun. Mag., vol. 48, no. 2, pp. 39-47, 2010.

[3] P. Polishuk, "Plastic optical fibers branch out," IEEE Commun. Mag., vol. 44, no. 9, pp. 140-148, 2006.

[4] D. O'Brien and M. Katz, "Optical wireless communications within fourth-generation wireless systems," J. Opt. Netw., vol. 4, pp. 312-322, 2005.

[5] T. Rokkas, T. Kamalakis, D. Katsianis, D. Varoutas, and T. Sphicopoulos, "Business prospects of wide-scale deployment of free space optical technology as a last-mile solution: A techno-economic evaluation," J. Opt. Netw., vol. 6, pp. 860-87, 2007.

[6] J. M. Kahn, W. J. Krause, and J. B. Carruthers, "Experimental characterization of non-directed indoor infrared channels," IEEE Trans. Commun., vol. 43, pp. 1613-1623, 1995.

[7] T. Komine and M. Nakagawa, "Integrated system of white LED visible-light communication and power-line communication," IEEE Trans. Consum. Electron., vol. 49, pp. 71-79, 2003.

[8] http://www.ict-omega.eu/.

[9] J. P. Javaudin, M. Bellec, P. Jaffré, A. Foglar, O. Hoffmann, and O. Isson, "Inter-MAC concept for gigabit home networks," presented at the IEEE 20th Int. Symp. on Personal, Indoor and Mobile Radio Communications, Tokyo, Japan, 2009.

[10] H. Le Minh, D. O'Brien, G. Faulkner, O. Bouchet, M. Wolf, and L. J. Grobe, "A 1.25-Gb/s indoor cellular optical wireless communications demonstrator," IEEE Photon. Technol. Lett., vol. 22, pp. 1598-1600, 2010.

[11] ICT-OMEGA Deliverable D4.2b, Physical Layer Design and Specification, Feb. 2010 [Online]. Available: http://www.ict-omega.eu/ fileadmin/documents/deliverables/Omega_D4.2b.pdf.

[12] A. Maiga, J. Y. Baudais, and J. F. Hélard, "Very high bit rate power line communications for home networks," in IEEE Int. Symp. on Power Line Communications and Its Applications, Dresden, Germany, 2009.

[13] G. Dede, D. Varoutas, T. Kamalakis, R. G. Fuentetaja, and J. P. Javaudin, "Evaluation of technological and socio-economic issues affecting the deployment of home networks: Evidence from the ICT-OMEGA project," Netnomics: Econ. Res. Electron. Netw., vol. 11, pp. 281-200, 2010.

[14] Y. Lee and K. A. Kozar, "Investigating the effect of website quality on e-business success: An analytic hierarchy process (AHP) approach," Decision Support Sys., vol. 42, pp. 1383-1401, 2006.

[15] F. T. S. Chan, H. K. Chan, H. C. W. Lau, and R. W. L. Ip, "An AHP approach in benchmarking logistics performance of the postal industry," Benchmarking: An International Journal, vol. 13, pp. 636-66, 2006.

[16] M. J. Liberatore and R. L. Nydick, "The analytic hierarchy process in medical and health care decision making: A literature review," Eur. J. Oper. Res., vol. 189, pp. 194-207, 2008.

[17] P. R. Drake, "Using the analytic hierarchy process in engineering education,” Int. J. Eng. Educ., vol. 14, pp. 191-196, 1998.

[18] M. C. Y. Tam and V. M. R. Tummala, "An application of the AHP in vendor selection of a telecommunications system," Omega, vol. 29, pp. 171-182, 2001.

[19] T. L. Saaty, "Decision-making with the AHP: Why is the principal eigenvector necessary," Eur. J. Oper. Res., vol. 145, pp. 85-91, 2003.

[20] I. Mollers, D. Jager, R. Gaudino, A. Nocivelli, H. Kragl, O. Ziemann, N. Weber, T. Koonen, C. Lezzi, A. Bluschke, and S. Randel, "Plastic optical fiber technology for reliable home networking: Overview and results of the EU project pof-all," IEEE Commun. Mag., vol. 47, no. 8, pp. 58-68, 2009.

[21] M. Kwiesielewicz and E. V. Uden, "Inconsistent and contradictory judgments in pairwise comparison method in the AHP," Comput. Oper. Res., vol. 31, pp. 713-719, 2004.

[22] N. Bhushan and K. Rai, Strategic Decision Making: Applying the Analytic Hierarchy Process. Springer, 2004.

[23] T. L. Saaty and L. G. Vargas, "Inconsistency and rank preservation," J. Math. Psychol., vol. 28, pp. 205-214, 1984.

[24] N. Gerdsri and D. F. Kocaoglu, "Applying the Analytic Hierarchy Process (AHP) to build a strategic framework for technology 
roadmapping," Math. Comput. Modell., vol. 46, pp. 1071-1080, 2007.

[25] D. H. Byun, "The AHP approach for selecting an automobile purchase model," Inf. Manage., vol. 38, pp. 289-297, 2001.

[26] C. W. Chang, C. R. Wu, C. T. Lin, and H. C. Chen, "An application of AHP and sensitivity analysis for selecting the best slicing machine," Comput. Ind. Eng., vol. 52, pp. 296-307, 2007.

[27] M. C. Y. Tam and V. M. R. Tummala, "An application of the AHP in vendor selection of a telecommunications system," Omega, vol. 29, pp. 171-182, 2001.

[28] D.-S. Ly-Gagnon, S. Tsukamoto, K. Katoh, and K. Kikuchi, "Coherent detection of optical quadrature phase-shift keying signals with carrier phase estimation," J. Lightwave Technol., vol. 24, pp. 12-21, 2006.

[29] G. Ntogari, T. Kamalakis, and T. Sphicopoulos, "Performance analysis of space time block coding techniques for indoor optical wireless systems," IEEE J. Sel. Areas Commun., vol. 27, pp. 1545-1552, 2009.

[30] B. T. Olsen and K. Stordahl, "Models for forecasting cost evolution of components and technologies," Telektronikk, vol. 4, pp. 138-148, 2004.

[31] J. P. Faure, "The IEEE P1901 project: Broadband over power lines," in Int. Conf. on Consumer Electronics, 2006, pp. 159-160.

Georgia Dede received her degree in informatics and telecommunications in 2005 and her M.Sc. in administration and economics of telecommunication networks in 2007 from the University of Athens, Greece. She is currently working on her Ph.D. at the same university. Since 2007, she has been an Adjunct Lecturer and Research Associate in the Department of Informatics and Telematics, Harokopio University of Athens.
Thomas Kamalakis obtained his B.Sc. in informatics, M.Sc. in telecommunications with distinction and Ph.D. in the field of integrated optics from the University of Athens in 1997, 1999 and 2004, respectively. Since 2005 he has been a Research Associate in the Optical Communications Laboratory of the University of Athens. In 2008, he was appointed Lecturer in the Department of Informatics and Telematics at the Harokopio University of Athens. Dr. Kamalakis is the author and co-author of more than 50 publications in scientific journals and conferences in the fields of optical communications and technoeconomics.

Dimitris Varoutas (M'98, SM'11) holds a physics degree and M.Sc. and Ph.D. diplomas in communications and technoeconomics from the University of Athens. He serves as an Assistant Professor in the Department of Informatics and Telecommunications at the University of Athens. He was involved in numerous European R\&D projects in the RACE I \&II, ACTS, Telematics, RISI and IST frameworks in the areas of telecommunications and technoeconomics. $\mathrm{He}$ actively participates in several technoeconomic activities for telecommunications, networks and services such the ICT-OMEGA and the CELTIC/CINEMA projects, as well as the conferences on Telecommunications TechnoEconomics. He also participates in or manages related national activities for technoeconomic evaluation of broadband strategies, telecommunications demand forecasting, price modeling, etc. His research interests span design of optical and wireless communications systems to technoeconomic evaluation of network architectures and services. He has published more than 80 publications in refereed journals and conferences in the area of telecommunications, optoelectronics and technoeconomics, including leading IEEE journals and conferences. $\mathrm{He}$ is a Senior Member of the Photonics (former LEOS), Communications, Education and Engineering Management Societies of the IEEE and serves as reviewer for several journals and conferences. Since 2007, he has been the Deputy Vice Chairman of ADAE, the National Authority for Communications Security and Privacy. 